\title{
Subtle Expressivity for Characters and Robots
}

\author{
Noriko Suzuki \\ ATR Media Information Science Laboratories \\ 2-2-2 Hikaridai, Seika, Soraku \\ Kyoto 619-0288, JAPAN \\ noriko@atr.co.jp
}

\author{
Christoph Bartneck \\ Technical University of Eindhoven \\ Den Dolech 2, 5600 MB Eindhoven \\ The Netherlands \\ christoph@bartneck.de
}

\section{SUBTLE EXPRESSIVITY}

People, both consciously and unconsciously, use subtle expressions to indirectly communicate their emotions and intentions through variations of the gaze direction, voice tone and gesture speed. People also perceive changes in the internal states of others from subtle changes in their expressivities while interacting with them. Subtle expressivity plays the supporting part to the leading role of explicit expressivity, such as contents of speech or category of facial expressions. However, subtle expressivity plays an important role to give moderate effects or gently regulate the relationship among the participants through a continuous interaction.

These subtle expressivities are little focused on the design of interactive media in the context of software products and computers. Only in the area of computer games in which pre-designed animated characters are used, the full potential of subtle expressivity is fully understood and used.

Although the general interest of the human-computer interaction research community in life-likeness and personality as a goal of software design is growing for reducing cognitive load [1,2], we are far from having coherent understanding of what subtle expressivity actually is and how products and processes can address it. We might question whether designing for subtle expressivity will result in gentle emotional effects on people and whether the processes and topics involved differ in any significant way from designing for believability or personality.

\section{CHALLENGES FOR SUBTLE EXPRESSIVITY}

As a result of the rapid progress of technology, computers have become interactive partners rather than useful tools for people. In the past ten years, the CHI community had a strong interest in studying the believability, life-likeness and personality generation for interactive media, such as animated characters and communication robots.

Copyright is held by the author/owner(s).

CHI 2003, April 5-10, 2003, Ft. Lauderdale, Florida, USA.

ACM 1-58113-637-4/03/0004
Most studies, however, focus on explicit expressions using non-verbal information including facial expression [3], nod and glance $[4,5]$ or para-linguistic features including speech style [6] or voice tone $[7,8]$. These expressions are designed to produce consistent emotions and intensions. So far, the subtle expressivity is only of peripheral interest compared to the interest in explicit expressivity.

One reason might be that it is even hard in face-to-face interaction among people to observe and define subjectively the subtle expressivities. Even if people misinterpreted a subtle expression of their communication partner, they still continue to interact and try to interpret further expressions.

The possibly best-known real world example for subtle expressivity is the facial expressions of the Mona Lisa (see Figure 1). Various interpretations of her smile are available and thoroughly discussed. The discussion can even be extended by including the expressions that her observers make in face-to-face contact.

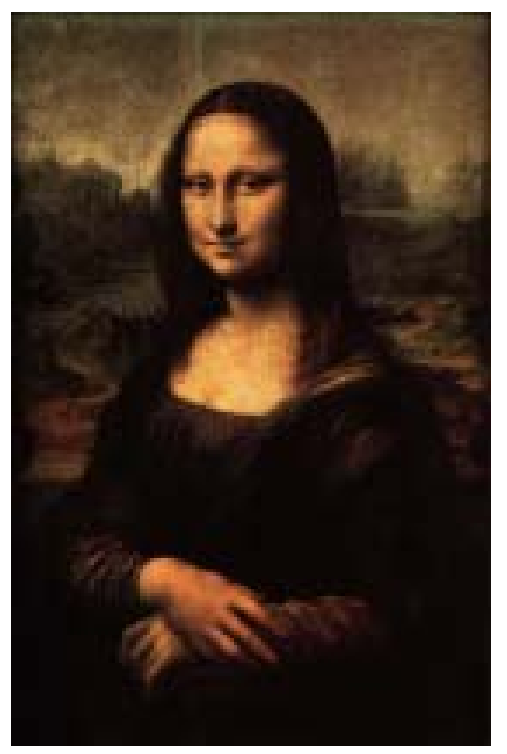

Figure 1: Mona Lisa by Leonardo Da Vinci.

Unfortunately, there is no clear tradition in either cognitive psychology or emotion psychology about the human abilities in using subtle expressivity in their various communication channels. Many researchers focus on deciding and categorizing unique emotional codes for 
explicit expressivity, such as an angry face, an OK sign or a happy voice.

\section{IMPORTANCE OF SUBTLE EXPRESSIVITY}

Conventional computer systems are constructed to help and assist with the user's work. However, recent applications are extending their focus to the area of entertainment and amusement. Electric toys and entertainment robots may soon invade our everyday lives. Subtle expressivity is a key requirement for the social acceptance of these types of characters and robots. The growing interest of researchers and designers may turn subtle expressivity into major issues for future interactive technology for both the office and the home.

The concepts and theories of subtle expressions are also required for product designers and developers. However, as mentioned the above section, they are not clarified in detail. If these concepts are arranged clearly, researchers and designers are going to be pioneers by creating useful guidelines for the development of interactive technology.

The measuring or evaluation methods for subtle expressivity are necessary for researchers and designers to support the development of these guidelines and to clearly identify the moderate effects of subtle expressivity. The proposal of new measuring methods beyond usability will also have an impact on cognitive science and psychology.

\section{GOAL OF THE WORKSHOP}

The workshop aims to provide a forum to discuss emerging issues in the design of subtle expressivities and its applications. Furthermore, we want to identify major issues involved in the interaction of subtle expressions and their effect on the user and discuss the role of cognitive science and psychology in the use of subtle expressions in the design of embodied characters.

We plan to cover the following topics:

Concepts, theories and guidelines: Conceptual contribution for design of subtle expressions including psychological theory or software quality models.

- How to create emotional expressions in the various communication channels?

- What kind of vocabulary of emotional and conversational expressions does the character need?

- What minimal set of subtle expressions is necessary to realize life-likeness of characters?

Implementation methods: Case study for software/hardware products using subtle expressivity.

- How can technology adapt to user preferences?

- What types of software architectures are necessary for an emotional expressive character?
- What subtle expressions to software architecture are important in designing characters and robots?

Measuring methods: Evaluation contribution of moderate effects of subtle expressions.

- How can these effects be measured?

- On what attributes of the interaction between the character and the user will the expressions have effects?

- How can subtle expressions enhance empathic interaction?

\section{DISSEMINATION}

We have a plan to edit a special issue of this workshop in ACM Transactions on Computer-Human Interaction or the International Journal of Human-Computer Studies. We will select the best papers and ask their authors to submit an extended version for a second review round.

\section{REFERENCES}

1. Oren, T., Salomon, G., Kreitman, K. and Don, A. 1990. Guides: characterizing the interface. In Laurel, B. Eds. The art of human-computer interface design. AddisonWesley.

2. Maes, P. 1994. Agents that reduce work and information overload. Communication of the ACM, 37, 7, pp. $30-40$.

3. Cohen, M.M., Beskow, J. and Massaro, D.W. 1998. Recent developments in facial animation: an inside view. In Proc. of AVSP' 98.

4. Yokoyama, M., Aoyama, K., Kikuchi, H. and Shirai, K. 1998. Use of non-verbal information in communication between human and robot. In Proc. of ICSLP'98.

5. Cassell, J. and Thorisson, K. R. 1999. The power of a nod and a glance: envelope vs. emotional feedback in animated conversational agents. Applied Artificial Intelligence, 13, 4-5, pp. 519-538.

6. Nass, C., Moon, Y. Fogg, B.J., Reeves, B. and Dryer, D.C. 1995. Can computer personalities be human personalities? Human-Computer Studies, 43, pp. 223239.

7. Hayashi, M. Ueda, H. and Kurihara, T. 1999. TVML (TV program Making Language) - automatic TV program generation from text-base script -, In Proc. of Imagina'99.

8. Nass, C. and Lee, K.M. 2000. Does computer-generated speech manifest personality? In Proc. of CHI2000, pp. 329-336. 